\title{
First Measurement of Antikaon Phase-Space Distributions in Nucleus-Nucleus Collisions at Subthreshold Beam Energies
}

\author{
M. Menzel ${ }^{d}$, I. Böttcher ${ }^{d}$, M. Dębowski ${ }^{e}$, F. Dohrmann ${ }^{f, \S}$, A. Förster ${ }^{b}$, E. Grosse $^{f, g}$, \\ P. Koczoń ${ }^{a}$, B. Kohlmeyer ${ }^{d}$, F. Laue ${ }^{a, *}$, L. Naumann ${ }^{f}$, H. Oeschler ${ }^{b}$, F. Pühlhofer ${ }^{d}$,

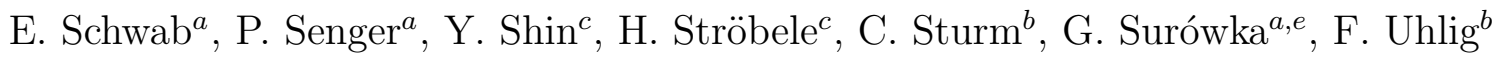 \\ A. Wagner ${ }^{f}$, W. Waluśe \\ (KaoS Collaboration) \\ ${ }^{a}$ Gesellschaft für Schwerionenforschung, D-64220 Darmstadt, Germany \\ ${ }^{b}$ Technische Universität Darmstadt, D-64289 Darmstadt, Germany \\ ${ }^{c}$ Johann Wolfgang Goethe Universität, D-60325 Frankfurt am Main, Germany \\ ${ }^{d}$ Phillips Universität, D-35037 Marburg, Germany \\ e Jagiellonian University, PL-30059 Kraków, Poland \\ ${ }^{f}$ Forschungszentrum Rossendorf, D-01314 Dresden, Germany \\ $g$ Technische Universität Dresden, D-01062 Dresden, Germany \\ $\S$ Present address: Argonne National Laboratory, Argonne, IL 60439, USA \\ * Present address: Ohio State University, Columbus, OH 43210, USA \\ PACS numbers: 25.75.Dw
}

\begin{abstract}
Differential production cross sections of $\mathrm{K}^{-}$and $\mathrm{K}^{+}$mesons have been measured as function of the polar emission angle in $\mathrm{Ni}+\mathrm{Ni}$ collisions at a beam energy of $1.93 \mathrm{AGeV}$. In near-central collisions, the spectral shapes and the widths of the rapidity distributions of $\mathrm{K}^{-}$and $\mathrm{K}^{+}$mesons are in agreement with the assumption of isotropic emission. In non-central collisions, the $\mathrm{K}^{-}$ and $\mathrm{K}^{+}$rapidity distributions are broader than expected for a single thermal source. In this case, the polar angle distributions are strongly forwardbackward peaked and the nonisotropic contribution to the total yield is about
\end{abstract}


one third both for $\mathrm{K}^{+}$and $\mathrm{K}^{-}$mesons. The $\mathrm{K}^{-} / \mathrm{K}^{+}$ratio is found to be about 0.03 independent of the centrality of the reaction. This value is significantly larger than predicted by microscopic transport calculations if in-medium modifications of $\mathrm{K}$ mesons are neglected. 
The behavior of strange particles in dense nuclear matter plays a crucial role in the dynamics of supernovae and for the stability of neutron stars 10 . The creation of negatively charged kaons in equilibrated neutron star matter becomes energetically possible if the electro-chemical potential exceeds the effective mass of kaons in the dense medium. It was speculated that a Bose condensation of $\mathrm{K}^{-}$mesons significantly softens the equation-of-state of neutron star matter and hence catalyzes the formation of low-mass black holes [2]. This idea is based on theoretical calculations predicting in-medium kaon-nucleon potentials which are repulsive for kaons but attractive for antikaons [5 8 .

Experiments with heavy-ion beams provide the unique possibility to study the creation and propagation of strange particles in baryonic matter at densities well above nuclear matter density. In nucleus-nucleus collisions both the total production cross sections and the angular distributions of strange mesons should be affected by the predicted in-medium potentials. The sensitivity on in-medium effects is enhanced at beam energies below the kaon production threshold in nucleon-nucleon collisions $(1.58 \mathrm{GeV}$ and $2.5 \mathrm{GeV}$ for the processes $\mathrm{NN} \rightarrow \mathrm{K}^{+} \Lambda \mathrm{N}$ and $\mathrm{NN} \rightarrow \mathrm{K}^{-} \mathrm{K}^{+} \mathrm{NN}$, respectively). The creation of strange mesons is dominated by secondary processes (like pion-nucleon collisions) which happen more frequently at higher densities [6,9, [0].

In recent years, data on kaon and antikaon production in nucleus-nucleus collisions at subthreshold beam energies have been collected at the heavy-ion synchrotron SIS at GSI in Darmstadt. The most remarkable findings are that (i) the $\mathrm{K}^{-}$yield is surprisingly high [11 13], (ii) the in-plane flow of $\mathrm{K}^{+}$mesons is vanishingly small in $\mathrm{Ni}+\mathrm{Ni}$ collisions at $1.93 \mathrm{AGeV}$ [14, (iii) preferential out-of-plane emission of $\mathrm{K}^{+}$mesons is observed in $\mathrm{Au}+\mathrm{Au}$ collisions at $1 \mathrm{AGeV}$ [15] and (iv) the spectral slope of $\mathrm{K}^{-}$mesons is steeper than the slope of $\mathrm{K}^{+}$mesons in $\mathrm{C}+\mathrm{C}$ collisions at $1.8 \mathrm{AGeV}$ [13]. These observations have all been explained as being due to modifications of the $\mathrm{K}$ meson properties in the nuclear medium [9, 10, 16 18].

In this Letter we report on the measurement of $\mathrm{K}^{-}$and $\mathrm{K}^{+}$rapidity and angular distributions in $\mathrm{Ni}+\mathrm{Ni}$ collisions at a beam energy of $1.93 \mathrm{AGeV}$. This energy is close to (below) the production threshold for $\mathrm{K}^{+}\left(\mathrm{K}^{-}\right)$mesons in nucleon-nucleon collisions. For the first 
time full rapidity distributions of $\mathrm{K}^{+}$and $\mathrm{K}^{-}$mesons become available at SIS energies. The production of $\mathrm{K}^{+}$mesons around target rapidity in central $\mathrm{Ni}+\mathrm{Ni}$ collisions at $1.93 \mathrm{AGeV}$ was already studied by the FOPI collaboration [19].

The experiment was performed with the Kaon Spectrometer (KaoS) at the heavy-ion synchrotron at GSI in Darmstadt [20]. This magnetic spectrometer has a large acceptance in solid angle and momentum $\left(\Omega \approx 30 \mathrm{msr}, p_{\max } / p_{\min } \approx 2\right)$. Kaon decays in flight are minimized by the short distance of $5-6.5 \mathrm{~m}$ from target to focal plane. Separate measurements of velocity, momentum and time-of-flight provide particle identification and allow for an online rejection of events with only pions and protons in the spectrometer by factors of $10^{2}$ and $10^{3}$, respectively. The background due to spurious tracks and pile-up is strongly reduced by trajectory reconstruction based on three large-area multi-wire chambers. The resulting signal-to-background ratio for $\mathrm{K}^{+}$and $\mathrm{K}^{-}$mesons is about 5:1 and 10:1, respectively. The total efficiency of the trigger, of the track recognition and of detection amounts to about $75 \%$, both for $\mathrm{K}^{+}$and $\mathrm{K}^{-}$mesons. The loss of kaons decaying in flight is determined (and corrected) by Monte Carlo simulations.

The ${ }^{58} \mathrm{Ni}$ beam had an intensity of up to $3 \times 10^{8}$ ions per spill ( 8 seconds long) and impinged on a ${ }^{58} \mathrm{Ni}$ target of $0.8 \mathrm{~mm}$ thickness. The beam intensity was similar for the $\mathrm{K}^{+}$ and $\mathrm{K}^{-}$experiments and for the different angle settings. The measurement was performed under four laboratory angles $\theta_{\text {lab }}=32^{\circ}, 40^{\circ}, 50^{\circ}$ and $60^{\circ}$ with 3 magnetic field settings accepting a momentum range of $280 \mathrm{MeV} / \mathrm{c}<p_{\text {lab }}<1120 \mathrm{MeV} / \mathrm{c}$. The corresponding phase space covered by the experiment ranges in transverse momentum from about $p_{t}=$ $150 \mathrm{MeV} / \mathrm{c}$ to $700 \mathrm{MeV} / \mathrm{c}$ and in rapidity from about $\mathrm{y}_{C M}=-0.65$ to +0.2 . The rapidity is defined as $\mathrm{y}_{C M}=\mathrm{y}_{l a b}-0.5 \times \mathrm{y}_{\text {proj }}$ with $\mathrm{y}_{\text {proj }}$ the projectile rapidity (in the laboratory) and $\mathrm{y}_{\text {lab }}=0.5 \times \ln \left(\left(E+p_{z}\right) /\left(E-p_{z}\right)\right)$. Here, $\mathrm{E}$ is the particle energy and $p_{z}$ is the particle momentum along the beam axis in the laboratory. The value $\mathrm{y}_{C M}=0$ corresponds to midrapidity and $\mathrm{y}_{C M}= \pm 0.89$ is target or projectile rapidity.

Approximatelly $60000 \mathrm{~K}^{+}$mesons and $8000 \mathrm{~K}^{-}$mesons have been registered. The large number of identified $\mathrm{K}$ mesons allowed the determination of differential cross sections in 
small intervalls of rapidity and transverse momentum. The event ensemble is subdivided in near-central and non-central reactions. The centrality of the collision is determined via the multiplicity of charged particles measured in the interval $12^{\circ}<\theta_{\text {lab }}<48^{\circ}$ by a hodoscope consisting of 84 plastic-scintillator modules. These particles - mostly protons and pions - have participated in the reaction and are therefore emitted to large angles. From a measurement with a minimum bias trigger the reaction cross section has been determined for each bin of charged particle multiplicity. As near-central collisions we define the most central $620 \pm 30 \mathrm{mb}$ of the reaction cross section which corresponds geometrically to impact parameters smaller than $4.4 \mathrm{fm}$.

Figure 1 shows the cross sections for $\mathrm{K}^{+}$(upper panels) and $\mathrm{K}^{-}$production (lower panels) in near-central (left) and non-central (right) collisions as function of the transverse kinetic energy $m_{T}-m$ for five equally wide bins in rapidity as defined in the lower right panel. The transverse mass is given by $m_{T}=\left(p_{T}^{2}+m^{2}\right)^{(1 / 2)}$ with $p_{T}$ the transverse momentum and $m$ the rest mass of the $\mathrm{K}$ mesons. The spectral yields are scaled by factors of 10 (see upper left panel). The error bars shown are due to statistics only. A systematic error of $10 \%$ due to efficiency corrections and normalization procedures has to be added to each data point. The lines represent Boltzmann distributions $d^{3} \sigma / d p^{3} \propto \exp \left(-m_{T} / T\right)$ fitted to the spectra individually for each rapidity bin above $\mathrm{y}_{C M}=-0.54$. The slope parameters of the spectra measured at $-0.69<\mathrm{y}_{C M}<-0.54$ are determined by linear extrapolation of the fitted $T$-values. The resulting values for $T$ are listed in Table 1.

In Figure 2 we show the multiplicity density distributions $\mathrm{dN} / \mathrm{dy}$ of $\mathrm{K}^{+}$and $\mathrm{K}^{-}$mesons for near-central (left panel) and non-central (right panel) collisions as function of the rapidity $\mathrm{y}_{C M}$. The particle multiplicity is given by $\mathrm{N}=\sigma_{K} / \sigma_{R}$. The inclusive production cross section $\sigma_{K}$ is obtained by integration of the spectra presented in Figure 1 using the Boltzmann fits to extrapolate to the unmeasured transverse mass regions. The measured fraction of the multiplicity density varies with rapidity. The values are given in Table 1 . In total, about $70 \%$ of the integrated multiplicity density distribution has been measured (taking advantage of the symmetry with respect to midrapidity). The error bars of the $\mathrm{dN} / \mathrm{dY}$ distributions 
shown in figure 2 include systematic uncertainties due to efficiency corrections and due to the extrapolation procedure. The uncertainties due to beam normalization and acceptance corrections (which affect all data points) amount to $7 \%$ and have to be added. The error bars of the $\mathrm{K}^{-} / \mathrm{K}^{+}$ratio do not include contributions from systematic uncertainties which affect both $\mathrm{K}^{+}$and $\mathrm{K}^{-}$data.

For the reaction cross section we use $\sigma_{R}=620 \mathrm{mb}$ for near-central collisions (as measured, see above) and $\sigma_{R}=\sigma_{g e o}-620 \mathrm{mb}$ for non-central collisions. The geometrical reaction cross section is defined as $\sigma_{\text {geo }}=4 \pi\left(\mathrm{r}_{0} \mathrm{~A}^{1 / 3}\right)^{2}$. For $\mathrm{Ni}+\mathrm{Ni}$ collisions we use a radius parameter of $\mathrm{r}_{0}=1.24 \mathrm{fm}$ 21] and obtain a value of $\sigma_{g e o}=2.9 \mathrm{~b}$. The inclusive cross sections for the production of $\mathrm{K}^{+}$and $\mathrm{K}^{-}$mesons in $\mathrm{Ni}+\mathrm{Ni}$ collisions at $1.93 \mathrm{AGeV}$ are $\sigma_{K^{+}}=87 \pm 10 \mathrm{mb}$ and $\sigma_{K^{-}}=2.8 \pm 0.4 \mathrm{mb}$ corresponding to a mean multiplicity of $\mathrm{N}_{K^{+}} \approx 3 \times 10^{-2}$ and $\mathrm{N}_{K^{-}} \approx$ $1 \times 10^{-3}$, respectively. The quoted errors include all systematic uncertainties.

The $\mathrm{K}^{+}$multiplicity density distribution for near-central collisions shown in Figure 2 can be compared to $\mathrm{K}^{+}$data measured by the FOPI collaboration in central $\mathrm{Ni}+\mathrm{Ni}$ collisions at $1.93 \mathrm{AGeV}$ around target rapidity [19]. The FOPI data were analyzed for the most central $350 \mathrm{mb}$ of the cross section corresponding to impact parameters below $3.3 \mathrm{fm}$ within a sharp cutoff model. The range of rapidities covered by the experiments FOPI and KaoS overlap only in a narrow region around $\mathrm{y}_{C M}=-0.6$. Here the measured $\mathrm{K}^{+}$multiplicity densities agree with each other within the error bars which are about $28 \%$ (FOPI) and 12\% (KaoS) including systematic uncertainties.

The solid lines in Figure 2 correspond to isotropic thermal distributions. The widths of the curves are given by the inverse slope parameters of the spectra measured around midrapidity (see Figure 1). For near-central Ni+Ni collisions, the measured dN/dy distributions of $\mathrm{K}^{+}$and $\mathrm{K}^{-}$mesons do not deviate significantly from isotropy. For non-central collisions, however, the widths of the multiplicity density distributions are clearly broader than expected for an isotropic thermal source both for $\mathrm{K}^{+}$and $\mathrm{K}^{-}$mesons.

The lower panel in Figure 2 presents the $\mathrm{K}^{-} / \mathrm{K}^{+}$multiplicity ratio as function of the rapidity for near-central (left) and non-central (right) $\mathrm{Ni}+\mathrm{Ni}$ collisions at $1.93 \mathrm{AGeV}$. The 
average values for the $\mathrm{K}^{-} / \mathrm{K}^{+}$ratios are $0.031 \pm 0.005$ both for near-central and non-central collisions. Transport calculations performed by two different groups predict a value of about 0.01 for the $\mathrm{K}^{-} / \mathrm{K}^{+}$ratio in central $\mathrm{Ni}+\mathrm{Ni}$ collisions at $1.93 \mathrm{AGeV}$ when taking into account bare (vacuum) masses for the K mesons [22,23]. These calculations include secondary production processes like $\pi \mathrm{N} \rightarrow \mathrm{K}^{+} \mathrm{K}^{-} \mathrm{N}$ and strangeness exchange reactions $\pi \Lambda \rightleftharpoons \mathrm{K}^{-} \mathrm{N}$. The measured $\mathrm{K}^{-} / \mathrm{K}^{+}$ratios can be reproduced by microscopic transport calculations by choosing appropriate values for the strengths of the repulsive $\mathrm{K}^{+} \mathrm{N}$ potential and for the attractive $\mathrm{K}^{-} \mathrm{N}$ potential which are in principle free parameters in those models. Up to now, predictions either overestimate [22] or underestimate [23] the measured $\mathrm{K}^{-} / \mathrm{K}^{+}$ratio shown in Figure 2 .

In a previous experiment we have studied the inclusive production of $\mathrm{K}^{+}$and $\mathrm{K}^{-}$mesons in $\mathrm{C}+\mathrm{C}$ collisions at $2 \mathrm{AGeV}$ [13]. The reabsorption of $\mathrm{K}^{-}$mesons in this light system via the strangeness exchange reaction $\mathrm{K}^{-} \mathrm{N} \rightarrow \mathrm{Y} \pi$ (with $\left.\mathrm{Y}=\Lambda, \Sigma\right)$ 24 should be much smaller than for the $\mathrm{Ni}+\mathrm{Ni}$ system which is almost 5 times heavier (the $\mathrm{K}^{+}$mesons cannot be absorbed). Nevertheless, the observed values for the $\mathrm{K}^{-} / \mathrm{K}^{+}$ratio were found to be very similar $\left(\mathrm{K}^{-} / \mathrm{K}^{+}\right.$ $=0.038 \pm 0.018$ for $\mathrm{C}+\mathrm{C}$ at $2 \mathrm{AGeV}$ ). This observation indicates that the losses of $\mathrm{K}^{-}$mesons in the $\mathrm{Ni}+\mathrm{Ni}$ system due to reabsorption are compensated by an enhanced production. It is interesting to note that in near-central $\mathrm{Ni}+\mathrm{Ni}$ collisions the $\mathrm{K}^{-} / \mathrm{K}^{+}$ratio is independent of transverse momentum whereas in non-central $\mathrm{Ni}+\mathrm{Ni}$ and in inclusive $\mathrm{C}+\mathrm{C}$ collisions 13 the $\mathrm{K}^{-} / \mathrm{K}^{+}$ratio decreases with increasing transverse momentum.

In order to quantify the deviation from isotropical $\mathrm{K}$ meson emission in the $\mathrm{Ni}+\mathrm{Ni}$ system we have calculated the ratio $R=\sigma_{i n v}\left(E_{C M}, \theta_{C M}\right) / \sigma_{i s o}\left(E_{C M}\right)$ with $\sigma_{i n v}$ the measured invariant kaon production cross section and $\sigma_{i s o}$ a thermal isotropic distribution. The function $\sigma_{i s o} \propto \exp \left(-E_{C M} / T\right)$ is fitted to the data separately for $\mathrm{K}^{+}$and $\mathrm{K}^{-}$and for near-central and non-central collisions. In order to determine an polar emission pattern which is representative for the bulk of the kaons, the values of $R$ are weighted with the kaon production cross section at a given $E_{C M}$. Then, the $R$-values which fall into one bin of $\theta_{C M}$ are averaged. The resulting distributions $R\left(\cos \theta_{C M}\right)$ are shown in Figure 3 for $\mathrm{K}^{+}$(upper panel) and $\mathrm{K}^{-}$ 
(lower panel) and for near-central (left) and non-central collisions (right).

The dashed lines represent the function $R\left(\cos \theta_{C M}\right) \propto 1+a_{2} \cdot \cos ^{2}\left(\theta_{C M}\right)$ which is fitted to the experimental distributions. The anisotropy factors $a_{2}$ are listed in Table 2 together with the values for the anisotropic fraction of the total yield. The total yield is proportional to the integral

$$
\int_{-1}^{+1} R\left(\cos \theta_{C M}\right) d \cos \theta_{C M} \propto\left(1+a_{2} / 3\right)
$$

and hence the nonisotropic contribution is calculated by $\left(a_{2} / 3\right) /\left(1+a_{2} / 3\right)$. The data in Figure 3 and the result of their analysis show that in near-central collisions the $\mathrm{K}^{-}$mesons were emitted isotropically whereas the emission of $\mathrm{K}^{+}$mesons is slightly enhanced at forwardbackward angles. In non-central collisions, however, the emission patterns of both $\mathrm{K}^{-}$and $\mathrm{K}^{+}$mesons are strongly forward-backward peaked. In these cases the nonisotropic contributions contain approximately one third of the total yield.

The phase-space distributions of kaons and antikaons presented in this letter constitute a detailed dataset on strange meson production in heavy-ion collisions at SIS energies. When confronted with transport calculations, this dataset offers the possibility to test the model assumptions in order to improve our understanding of the in-medium properties of hadrons.

In summary, we report the first measurement of $\mathrm{K}^{-}$and $\mathrm{K}^{+}$meson multiplicities as function of rapidity and polar angle in heavy-ion collisions at SIS energies. In near-central $\mathrm{Ni}+\mathrm{Ni}$ collisions, the spectral shapes and the widths of the multiplicity density distributions of $\mathrm{K}^{-}$and $\mathrm{K}^{+}$mesons are rather similar and in reasonable agreement with the assumption of isotropic emission. In non-central collisions, however, the $\mathrm{K}^{+}$and $\mathrm{K}^{-}$rapidity density distributions are broader than expected for isotropic emission from a single thermal source with a temperature given by the inverse slope parameter of the spectra measured around midrapidity. This effect is even more obvious in the polar angle distributions of $\mathrm{K}^{+}$and $\mathrm{K}^{-}$mesons which are strongly forward-backward peaked for non-central collisions. For both centrality selections, the $\mathrm{K}^{-} / \mathrm{K}^{+}$ratio is about 0.03 . This value is about three times larger than predicted by transport calculations in which in-medium modifications of $\mathrm{K}^{+}$and $\mathrm{K}^{-}$ 
mesons are neglected.

This work was supported by the German Federal Government (BMBF), by the Polish Committee of Scientific Research (Contract No. 2P03B11515) and by the GSI fund for University collaborations. 


\section{REFERENCES}

[1] N. K. Glendenning, F. Weber, S. A. Moszkowski, Phys. Rev. C 45 (1992) 844

[2] G.E. Brown and H.A. Bethe, Astrophys. Jour. 423 (1994) 659 and Nucl. Phys. A 567 (1994) 937

[3] G.Q. Li, C.H. Lee and G.E. Brown, Phys. Rev. Lett. 79 (1997) 5214

[4] H. Heiselberg and M. Hjorth-Jensen, Phys. Rep. 328 (2000) 237

[5] G.E. Brown, C.H. Lee, M. Rho and V. Thorsson, Nucl. Phys. A 567 (1994) 937

[6] J. Schaffner-Bielich, J. Bondorf, I. Mishustin, Nucl. Phys. A 625 (1997) 325

[7] T. Waas, N. Kaiser and W. Weise, Phys. Lett. B 379 (1996) 34

[8] M. Lutz, Phys. Lett. B 426 (1998) 12

[9] G.Q. Li, C.M. Ko, X.S. Fang, Phys. Lett. B 329 (1994) 149

[10] W. Cassing et al., Nucl. Phys. A 614 (1997) 415

[11] A. Schröter et al., Z. Phys. A 350 (1994) 101

[12] R. Barth et al., Phys. Rev. Lett. 78 (1997) 4007

[13] F. Laue, C. Sturm et al., Phys. Rev. Lett. 82 (1999) 1640

[14] J. Ritman et al., Z. Phys. A 352 (1995) 355

[15] Y. Shin et al., Phys. Rev. Lett. 81 (1998) 1576

[16] G.Q. Li et al., Phys. Rev. Lett. 74 (1995) 235 and Phys. Lett. B 381 (1996) 17

[17] G.Q. Li, C.M. Ko and G.E. Brown, Phys.Lett. B 381 (1996) 17

[18] E. Bratkovskaya, W. Cassing, U. Mosel, Phys. Lett. B 424 (1998) 244

[19] D. Best et al., Nucl. Phys. A 625 (1997) 307 
[20] P. Senger et al., Nucl. Instr. Meth. A 327 (1993) 393

[21] A. Wagner, PhD thesis, Tech. Univ. Darmstadt, 1996

[22] G.Q. Li and G.E. Brown, Phys. Rev. C 58 (1998) 1698

[23] W. Cassing and E. Bratkovskaya, Phys. Rep. 308 (1999) 65 and private communication

[24] C.B. Dover and G.E. Walker, Phys. Rep.89 (1982) 1 


\section{TABLES}

Table 1: Rapidity range $\mathrm{y}_{C M}$, inverse slope parameters $T$ and measured fraction $\mathrm{F}_{\text {meas }}$ of $K^{+}$and $\mathrm{K}^{-}$mesons in near-central and non-central $\mathrm{Ni}+\mathrm{Ni}$ collisions at $1.93 \mathrm{AGeV}$.

\begin{tabular}{|c|c|c|c|c|c|}
\hline \hline $\mathrm{y}_{C M}$ & $\begin{array}{r}\text { near-central } \\
T\left(\mathrm{~K}^{+}\right)(\mathrm{MeV})\end{array}$ & $\begin{array}{r}\text { near-central } \\
T\left(\mathrm{~K}^{-}\right)(\mathrm{MeV})\end{array}$ & $\begin{array}{r}\text { non-central } \\
T\left(\mathrm{~K}^{+}\right)(\mathrm{MeV})\end{array}$ & $\begin{array}{r}\text { non-central } \\
T\left(\mathrm{~K}^{-}\right)(\mathrm{MeV})\end{array}$ & $\mathrm{F}_{\text {meas }}$ \\
\hline$-0.69--0.54$ & $86 \pm 10$ & $84 \pm 15$ & $79 \pm 10$ & $67 \pm 10$ & $\approx 40 \%$ \\
$-0.54--0.39$ & $87 \pm 5$ & $79 \pm 6$ & $81 \pm 3$ & $70 \pm 7$ & $\approx 80 \%$ \\
$-0.39--0.24$ & $99 \pm 4$ & $100 \pm 6$ & $89 \pm 4$ & $73 \pm 7$ & $\approx 80 \%$ \\
$-0.24--0.09$ & $96 \pm 4$ & $106 \pm 11$ & $87 \pm 3$ & $84 \pm 5$ & $\approx 65 \%$ \\
$-0.09-+0.06$ & $107 \pm 7$ & $94 \pm 12$ & $97 \pm 6$ & $79 \pm 6$ & $\approx 40 \%$ \\
\hline \hline
\end{tabular}

Table 2: Anisotropy factor $a_{2}$ and nonisotropic fraction of the total yield $\mathrm{F}_{\text {noniso }}=$ $\left(a_{2} / 3\right) /\left(1+a_{2} / 3\right)$ for $\mathrm{K}^{+}$and $\mathrm{K}^{-}$mesons in near-central and non-central $\mathrm{Ni}+\mathrm{Ni}$ collisions at $1.93 \mathrm{AGeV}$.

\begin{tabular}{|c|c|c|c|c|}
\hline \hline & $\begin{array}{c}\text { near-central } \\
\mathrm{K}^{+}\end{array}$ & $\begin{array}{c}\text { near-central } \\
\mathrm{K}^{-}\end{array}$ & $\mathrm{K}^{+}$ & non-central \\
\hline$a_{2}$ & $0.6 \pm 0.2$ & $0.2 \pm 0.2$ & $1.6 \pm 0.2$ & $\mathrm{~K}^{-}$ \\
\hline $\mathrm{F}_{\text {noniso }}$ & $0.17 \pm 0.05$ & $0.06 \pm 0.06$ & $0.35 \pm 0.03$ & $1.7 \pm 0.4$ \\
\hline \hline
\end{tabular}


Figure 1: Production cross sections for $\mathrm{K}^{+}$(upper panel) and $\mathrm{K}^{-}$mesons (lower panel) in near-central (left) and non-central (right) $\mathrm{Ni}+\mathrm{Ni}$ collisions at $1.93 \mathrm{AGeV}$ as function of the transverse kinetic energy $m_{T}-m$. The data are sorted in bins of rapidity (as indicated in the lower right panel) and are scaled by factors of 10 (see upper left panel). The lines correspond to Boltzmann distributions fitted to the data (see text).

Figure 2: Multiplicity density distribution for K-mesons as function of rapidity in nearcentral (left) and non-central (right) $\mathrm{Ni}+\mathrm{Ni}$ collisions at $1.93 \mathrm{AGeV}\left(\mathrm{y}_{C M}=0\right.$ corresponds to midrapidity). Full data points are measured and mirrored at $\mathrm{y}_{C M}=0$ (open points). Upper panel: $\mathrm{K}^{+}$, middle panel $\mathrm{K}^{-}$, lower panel: $\mathrm{K}^{-} / \mathrm{K}^{+}$ratio. The $\mathrm{K}^{+}$and $\mathrm{K}^{-}$data for non-central collisions are multiplied by a factor of 4 . The lines correspond to isotropic thermal distributions with temperatures given by the inverse slope parameters of the spectra measured around midrapidity (T-values are indicated).

Figure 3: Polar angle distributions of $K^{+}$(upper panel) and $K^{-}$mesons (lower panel) in the center-of-mass system for near-central (left) and non-central (right) $\mathrm{Ni}+\mathrm{Ni}$ collisions at $1.93 \mathrm{AGeV}$. Full data points are measured and mirrored at $\mathrm{y}_{C M}=0$ (open points). The lines correspond to the function $R \propto 1+a_{2} \cdot \cos ^{2}\left(\theta_{C M}\right)$ fitted to the data (see text). The resulting values for $a_{2}$ are indicated. 


\section{FIGURES}

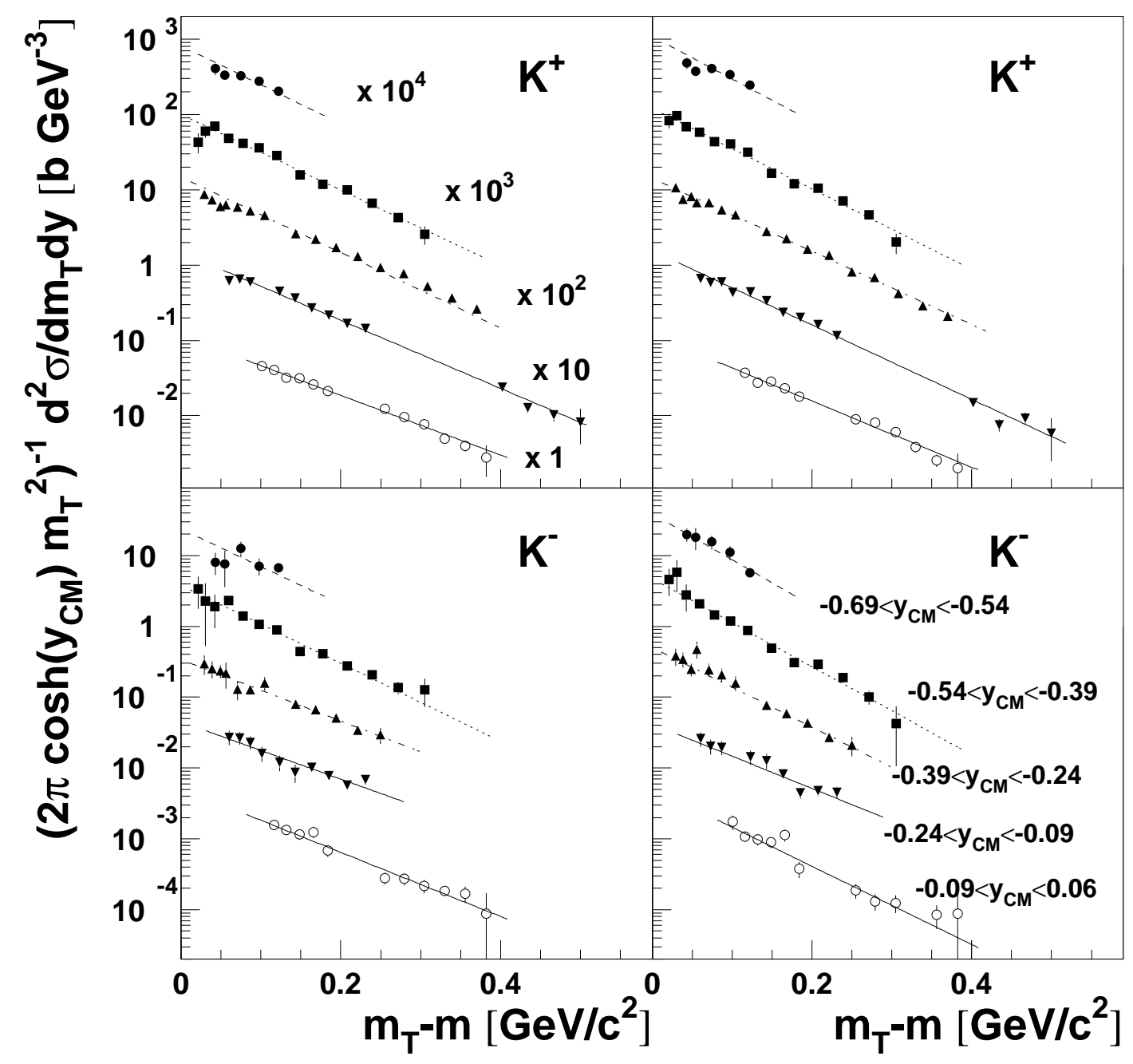

FIG. 1. 


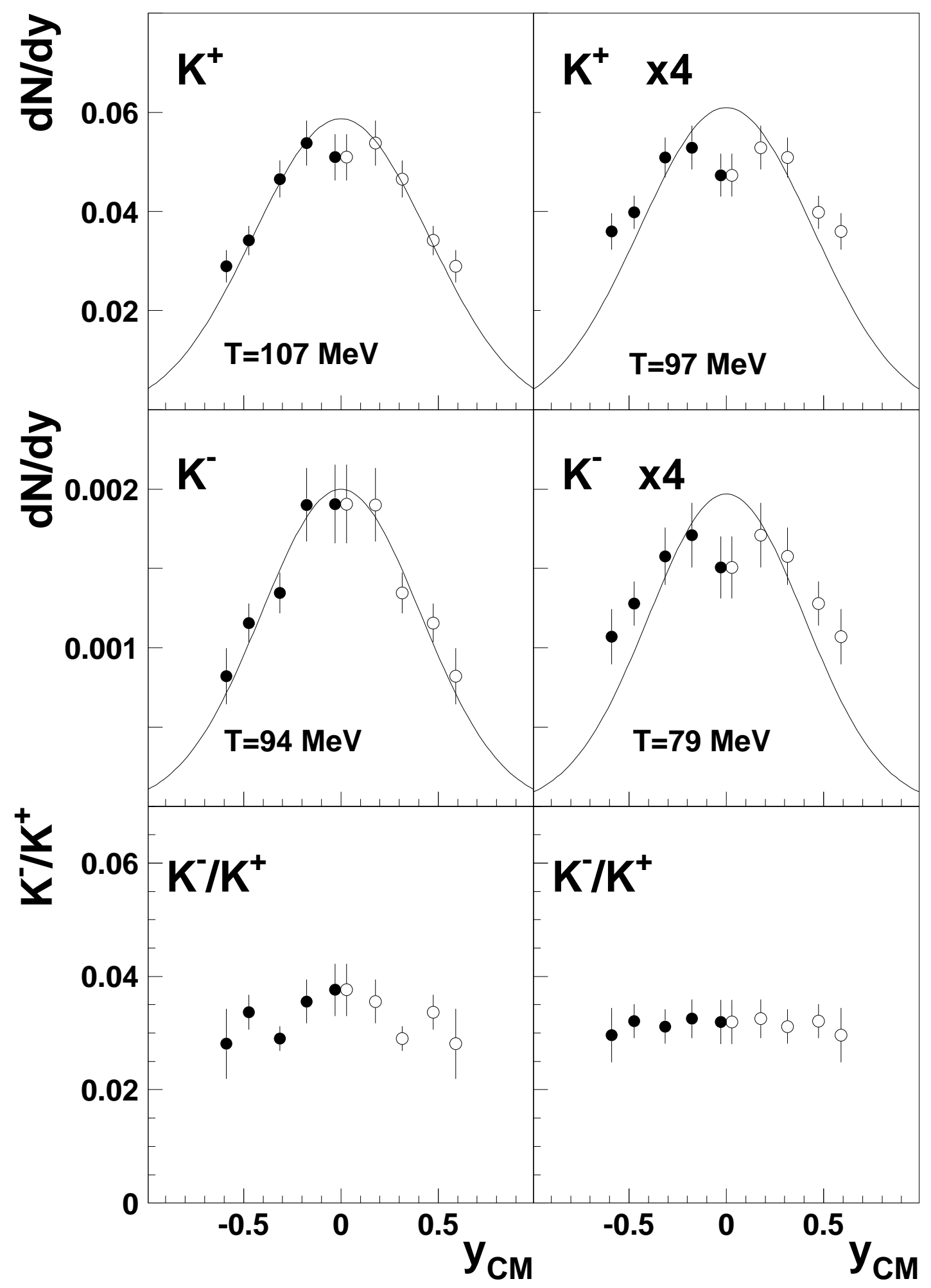


FIG. 2.

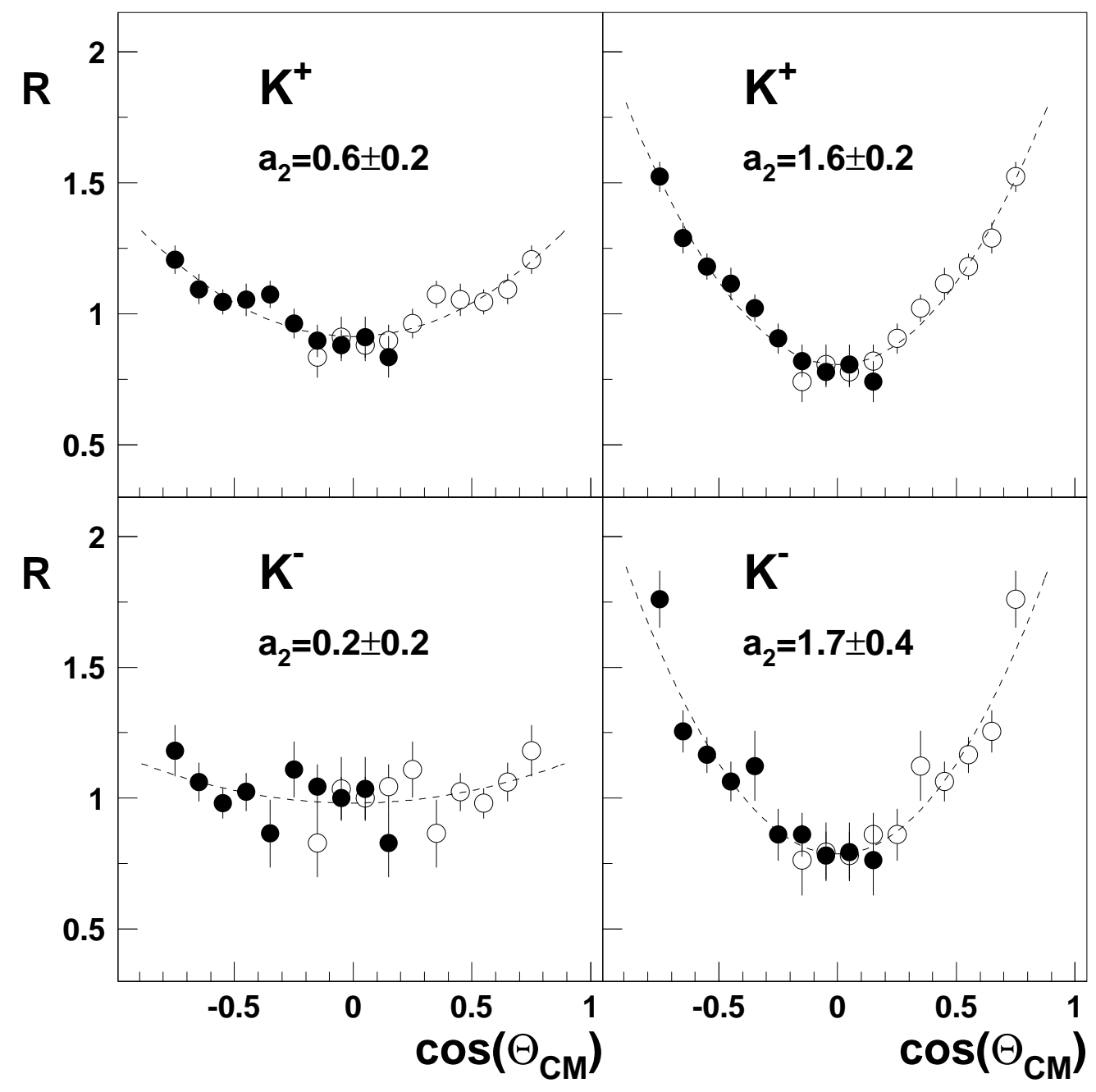

FIG. 3. 